\title{
Bibliometric analysis of global lithium ion battery research trends from 1993 to 2008
}

This article was published in the following Dove Press journal:

Reports in Electrochemistry

II November 20I I

Number of times this article has been viewed

\section{Lin Wei}

Jiayong Zhou

Xuhui Mao

School of Resource and

Environmental Science,

Wuhan University, Wuhan,

People's Republic of China
Correspondence: Xuhui Mao School of Resource and Environmental Science, Wuhan University, Wuhan City, Hubei Province 430079,

People's Republic of China

Tel +86 2768775799

Fax +86 2768775799

Email clab@whu.edu.cn
Abstract: Lithium ion battery research has experienced rapid growth over the last 16 years with regards to knowledge and technological innovation. In this paper, a bibliometric analysis was applied to evaluate the global scientific output of lithium ion battery research from 1993 to 2008 . The results show that a power model could be applied to demonstrate the high correlation between cumulative number of articles and publication year. Internal collaborative articles are prevalent in recent years and are predicted to increase in the future. Summaries on the most productive authors, corresponding authors, and institutes are presented. Researchers from North America, western Europe, and eastern Asia contributed most of the publications. Some findings on research trends are provided through a comprehensive analysis of author keywords and words in the title, which may facilitate the discussion on future hotspots in lithium ion battery research.

Keywords: lithium ion battery, bibliometric analysis, global trends

\section{Introduction}

Since the 1970s, owing to its lightweight, high voltage, and excellent energy density, lithium metal had been targeted for use in the assembly of primary lithium cells. However, lithium dendrite growth during each subsequent discharge-recharge cycle limited the application of rechargeable batteries utilizing metallic lithium anodes. Towards the end of the 1970s and early 1980s, Murphy et al ${ }^{1}$ firstly and then Scrosati et $\mathrm{al}^{2}$ demonstrated substitution of the metallic lithium anode by a second insertion material in order to address the safety issue surrounding the use of lithium metal, and developed what is now known today as lithium ion or "rocking-chair" technology. In 1991, the first commercial lithium ion battery based on a graphite anode and lithium cobalt oxide cathode was released by Sony. ${ }^{3}$ Since then, lithium ion batteries have gradually become the dominant secondary cells for portable electronics products, such as cellular phones, digital cameras, camcorders, handheld game machines, and portable audio players. The worldwide market size for lithium ion batteries (including lithium ion polymer batteries) was experiencing double-digit growth and reached 2.4 billion cells in $2007 .^{4}$

Meanwhile, the huge market demand has fueled scientific research of the lithium ion battery. Since the early 1990s, there has been an extraordinary amount of work on all aspects of lithium ion chemistry, and battery design, manufacture, and application. The annual number of papers on scientific production related to lithium ion battery research has grown from only 61 in 1993 to 950 in 2008. More specifically, the total number of publications had climbed to over 7000 papers in 2008. Interestingly, in spite 
of this rapid growth and productivity in the field, few have attempted to gather systematic data on the scale and scope of this explosive scientific inquiry. The panorama of global lithium ion battery research has never been described explicitly in a quantitative way in the previous literature.

As a tool to deal with the vast amount of literature, the bibliometric method has already been widely applied as an analysis technique for scientific production and research status in many disciplines of science and engineering. ${ }^{5,6}$ In the present study, the quality and evolution of lithium ion battery research from 1993 to 2008 have been quantitatively analyzed and assessed through improved bibliometric methods. Bibliometric information, such as times cited, affiliations, publication patterns, author keywords, and words in title were effectively utilized. This study aims to facilitate future discussion on developing trends in lithium ion battery research, therefore guiding researchers in their specific fields of science.

\section{Data and methodology}

Documents used in this study were derived from the Science Citation Index Expanded database of the Institute of Scientific Information. Documents, the topics of which meet the search criteria below, were downloaded from 1993 to 2008. Search terms were "Li-ion", "Li-ions", "Li ion", "Li ions", "lithium ion", "lithium ions", "lithium-ion", or "lithium-ions" and "cathode", "anode", "electrolyte", "negative electrode", "positive electrode", "battery", “cell”, "cathodes", "anodes", "electrolytes", "batteries", "cells", "negative electrodes", or "positive electrodes".

The total output was 9056 publications from 656 journals. Articles $(7025,77.57 \%)$ were the most frequent publication type, followed by proceedings papers $(1755,19.38 \%)$, reviews $(128,1.41 \%)$, letters $(46,0.51 \%)$, news items (32, 0.35\%), meeting abstracts $(30,0.33 \%)$, editorials $(17,0.19 \%)$, corrections $(16,0.18 \%)$, notes $(3)$, addition corrections (2), and reprints (2).

In this work, publications originating from England, Scotland, North Ireland, and Wales were grouped under the UK heading. Papers originating from Hong Kong were calculated individually, and separately from China. Collaboration type was determined by the addresses of the authors, where the term "independent" was assigned for the articles by a single institute. On the other hand, "collaboration" was assigned for papers with authors from more than one institute, and both internal and international collaborations included. The impact factor of a journal in this study was determined for each document as the Journal Citation Reports in the year 2008.
The times cited per publication (TCP) was used to denote the citation impact of a particular group of publications, which included both self-citation and independent citation.

\section{Results and discussion Publication output}

The publication output from 1993 to 2008 is listed in Table 1. The annual number of articles increased from the minimum 61 in 1993 to 950 in 2008. For the period from 1993 to 2008, the cumulative number of papers consistently increased (Figure 1), the progression of which was represented by two power models:

$$
\begin{array}{ll}
1993-1997 & y=58.218 x^{1.3353} \\
1997-2008 & y=13.075 x^{2.2475}
\end{array}
$$

The plot of the data revealed a high coefficient of correlation in the period 1993 to $1997\left(R^{2}=0.996\right)$ and 1997 to $2008\left(R^{2}=0.997\right)$. The publication growth rate in the later period was even higher than that of the period from 1993 to 1997. Moreover, due to the high coefficient of correlation, equation 2 can be used to estimate the number of future scientific articles related to lithium ion battery research. It can be predicted that, in 2015, the cumulative number of scientific articles on lithium ion battery research will be more than twice the number of publications in 2008.

\section{Publication performances: authorship, institutes, and countries}

Author address information was not available for $33(0.47 \%)$

articles in the Institute of Scientific Information, and 6992

Table I Annual production of research on lithium ion battery

\begin{tabular}{lll}
\hline Year & Publications $(\mathbf{n})$ & Cumulative (n) \\
\hline 1993 & 61 & 61 \\
1994 & 78 & 139 \\
1995 & 99 & 238 \\
1996 & 142 & 380 \\
1997 & 141 & 521 \\
1998 & 240 & 761 \\
1999 & 275 & 1036 \\
2000 & 299 & 1335 \\
2001 & 376 & 1711 \\
2002 & 471 & 2182 \\
2003 & 558 & 2740 \\
2004 & 652 & 3392 \\
2005 & 759 & 4151 \\
2006 & 924 & 5075 \\
2007 & 1000 & 6075 \\
2008 & 950 & 7025 \\
\hline
\end{tabular}




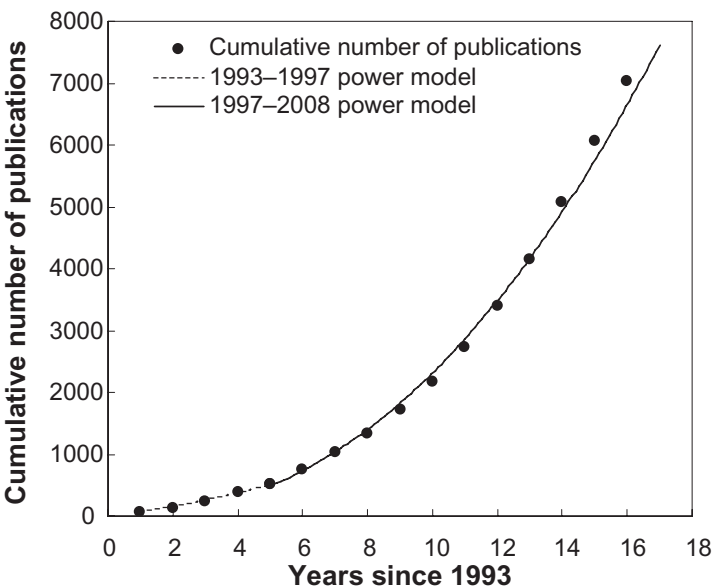

Figure I Cumulative number of publications by year.

articles were included in this analysis. In the 6992 papers published, there were 10,367 authors, amongst which 5965 authors (57.54\%) contributed to one paper, 1703 (16.43\%) to two papers, $788(7.60 \%)$ to three papers, $453(4.37 \%)$ to four papers, $328(3.16 \%)$ to five papers, 237 (2.29\%) to six papers, $147(1.42 \%)$ to seven papers, and $123(1.19 \%)$ to eight. Dahn at Dalhousie University in Canada contributed 153 papers, the highest number of papers published, followed by Chen and Wang from the Chinese Academy of Science, with 105 and 89 papers, respectively.

Additionally, the top 20 most prolific authors and most productive corresponding authors are shown in Table 2 .
Among the top 20 authors, nine authors were from China, and two were from Japan. As the corresponding authors on 42 articles, Dahn and He from Tsing Hua University of China both ranked the highest in the corresponding author analysis. The TCP rank can reveal the impact of a particular author's contribution in the development of lithium ion battery research. Tarascon from University Picardie Jules Verne, France, produced 66 articles and received the most citations per publication of these top 20 most prolific authors.

Table 3 depicts institutes with the largest number of publications and with the greatest citations per publication in lithium ion battery research during 1993-2008. The most prolific and often cited institutes were from the three large geographic regions (North America, western Europe, and eastern Asia). Five Chinese institutes were included in the top ten institutes with the greatest number of publications. By contrast, the top ten institutions with the greatest number of citations per publication contained four institutions from the United States but no institution from China. This phenomenon shows that while China has produced the greatest quantity of papers on lithium ion battery research, the quality of publications from institutions in China requires improvement.

Of all the 6992 articles, 1132 articles were international collaborative publications. These articles had an average TCP of 16.976, while the others from single countries

Table 2 The top twenty authors with the greatest number of papers in Li-ion battery research

\begin{tabular}{|c|c|c|c|c|c|}
\hline \multicolumn{2}{|c|}{ Rank } & \multirow[t]{2}{*}{$A U$} & \multirow[t]{2}{*}{ Institute } & \multirow{2}{*}{$\begin{array}{l}\text { Total articles } \\
\text { P/TCP }\end{array}$} & \multirow{2}{*}{$\frac{\text { CAU }}{\text { P/TCP }}$} \\
\hline AU & CAU & & & & \\
\hline $\mathrm{I}$ & I & Dahn, JR & Dalhousie Univ & $|53 / 4| .85$ & $42 / 28.86$ \\
\hline 2 & 24 & Chen, LQ & Chinese Acad Sci & $105 / 21.33$ & $19 / 13.05$ \\
\hline 3 & 7 & Wang, ZX & Chinese Acad Sci & $89 / 12.74$ & $30 / 10.50$ \\
\hline 4 & - & Wan, CR & Tsinghua Univ & $80 / 7.70$ & - \\
\hline 5 & 7 & Yoshio, M & Saga Univ & $76 / 24.70$ & $30 / 26.23$ \\
\hline 6 & - & Jiang, CY & Tsinghua Univ & $73 / 7.36$ & - \\
\hline 7 & 219 & Liu, HK & Univ Wollongong & $72 / 15.83$ & $5 / 21.40$ \\
\hline 7 & 9 & Zhao, XB & Zhejiang Univ & $72 / 6.04$ & $29 / 7.66$ \\
\hline 9 & 96 & Tarascon, JM & Univ Picardie Jules Verne & $66 / 51.49$ & $9 / 121.56$ \\
\hline 9 & 115 & Huang, XJ & Chinese Acad Sci & $66 / 22.94$ & $8 / 27.00$ \\
\hline 9 & 6 & Sun, YK & Hanyang Univ & $66 / 17.42$ & $34 / 16.53$ \\
\hline 12 & 13 & Aurbach, D & Bar Ilan Univ & $62 / 35.71$ & $26 / 41.85$ \\
\hline 13 & 13 & Scrosati, B & Univ Roma La Sapienza & $61 / 28.7 \mid$ & $26 / 19.08$ \\
\hline 14 & 431 & Cao, GS & Zhejiang Univ & $59 / 6.51$ & $3 / 9.00$ \\
\hline 15 & I & $\mathrm{He}, \mathrm{XM}$ & Tsinghua Univ & $58 / 5.31$ & $42 / 5.55$ \\
\hline 16 & 3 & Chen, $\mathrm{CH}$ & Univ Sci \& Technol China & $55 / 10.38$ & $40 / 9.73$ \\
\hline 17 & 32 & Amine, $\mathrm{K}$ & Argonne Natl Lab & $53 / 21.62$ & $|7 / 27.7|$ \\
\hline 17 & || $2 \mid$ & Ogumi, Z & Kyoto Univ & $53 / 15.93$ & $\mathrm{I} / 0$ \\
\hline 17 & 64 & Tirado, JL & Univ Cordoba & $53 / 9.43$ & $12 / 15.58$ \\
\hline 20 & 20 & Lee, JY & Natl Univ Singapore & $51 / 24.28$ & $20 / 26.20$ \\
\hline
\end{tabular}

Abbreviations: $\mathrm{CAU}$, corresponding author; $\mathrm{P}$, number of publications; TCP, citation time per publication. 
Table 3 The top ten institutes with the most number of papers and the top ten institutes with the greatest number of citations per publication in Li-ion battery research

\begin{tabular}{|c|c|c|c|c|c|c|c|c|}
\hline \multicolumn{2}{|l|}{ Rank } & \multirow[t]{2}{*}{ Institute } & \multirow[t]{2}{*}{$\mathbf{P}$} & \multirow[t]{2}{*}{ TCP } & \multicolumn{2}{|l|}{$\mathbf{P}$} & \multicolumn{2}{|l|}{ TCP } \\
\hline $\mathbf{P}$ & TCP & & & & IP & CP & IP & $\mathbf{C P}$ \\
\hline I & 705 & Chinese ACAD SCl & 286 & 11.14 & 180 & 106 & 12.63 & 8.60 \\
\hline 2 & 834 & Tsing Hua Univ & 146 & 9.25 & 87 & 59 & 7.41 & 11.97 \\
\hline 3 & 906 & Fu Dan Univ & 138 & 8.16 & 105 & 33 & 8.52 & 7.00 \\
\hline 4 & 175 & Dalhousie Univ & 137 & 32.20 & 90 & 47 & 39.14 & 18.89 \\
\hline 5 & 351 & Argonne Natl Lab & 133 & 20.11 & 47 & 86 & 20.87 & 19.70 \\
\hline 6 & 1155 & Zhejiang Univ & 132 & 5.88 & 98 & 34 & 6.88 & 3.00 \\
\hline 7 & 541 & Kyoto Univ & 120 & 14.33 & 43 & 77 & $|7.5|$ & 12.56 \\
\hline 8 & 1720 & Cent S Univ Technol & 108 & 1.76 & 56 & 52 & 1.66 & 1.87 \\
\hline 9 & 581 & Hanyang Univ & 101 & 13.31 & 15 & 86 & 5.40 & 14.69 \\
\hline 10 & 380 & Univ Calif Berkeley & 97 & 20.27 & 49 & 48 & 14.84 & 25.81 \\
\hline 1030 & I & Fuji Photo Film Co Ltd & I & 776.00 & - & 1 & - & 776.00 \\
\hline 1030 & I & Fuji Film Celltec & I & 776.00 & - & I & - & 776.00 \\
\hline 1030 & 3 & Ontario Canc Inst & I & 580.00 & - & I & - & 580.00 \\
\hline 1030 & 4 & Bell Commun Res Inc & 1 & 231.00 & - & 1 & - & 231.00 \\
\hline 353 & 5 & Colorado State Univ & 5 & 215.20 & 3 & 2 & 322.67 & 54.00 \\
\hline 699 & 6 & UPJV & 2 & 195.50 & - & 2 & - & 195.50 \\
\hline 1030 & 7 & UJF Grenoble & I & 192.00 & - & 1 & - & 192.00 \\
\hline 1030 & 8 & Vienna Univ Technol & I & 177.00 & - & I & - & 177.00 \\
\hline 1030 & 9 & FMC Corp & I & 175.00 & I & - & 175.00 & - \\
\hline 699 & 10 & Rayovac Corp & 2 & 148.50 & I & 1 & 104.00 & 193.00 \\
\hline
\end{tabular}

Abbreviations: CP, collaborative publications; IP, independent publications; P, number of publications; TCP, citation time per publication.

had a lower TCP of 13.959. Among the total 56 countries/ territories over the investigation period, 18 countries produced articles exclusively by way of international collaboration, while five countries produced no international collaborations at all in the lithium ion battery research. Nine countries contributed only one collaborative article. The top 20 highest producing countries are listed in Table 4. The United States had the most frequent partners, accounting for $41.61 \%$ of the international collaborative articles, followed by Japan with $19.79 \%$, France with $18.73 \%$. China, with the

Table 4 Top 20 countries or regions producing greatest number of papers in lithium ion battery research 1993-2008

\begin{tabular}{|c|c|c|c|c|c|c|c|}
\hline Country & $\mathbf{P}$ & $\begin{array}{l}\text { Rank } \\
\text { (\% of all P) }\end{array}$ & IP & $\begin{array}{l}\text { Rank } \\
\text { (\% of all IP) }\end{array}$ & CP & $\begin{array}{l}\text { Rank } \\
\text { (\% of all CP) }\end{array}$ & $\frac{\mathrm{TCP}}{\mathrm{IP} / \mathrm{CP}}$ \\
\hline China & 1855 & I (26.53) & 1683 & I (26.53) & 172 & $5(15.19)$ & $6.35 / 9.99$ \\
\hline US & 1517 & $2(21.70)$ & 1046 & $2(21.70)$ & 471 & I (4I.6I) & $20.19 / 21.02$ \\
\hline Japan & 1216 & 3 (I7.39) & 992 & 3 (I7.39) & 224 & 2 (19.79) & $16.19 / 16.64$ \\
\hline South Korea & 632 & $4(9.04)$ & 457 & $4(9.04)$ & 175 & $4(15.46)$ & $|3.25 /| 3.7 \mid$ \\
\hline France & 462 & $5(6.6 I)$ & 250 & $5(6.61)$ & 212 & $3(18.73)$ & $17.00 / 20.23$ \\
\hline Canada & 279 & 6 (3.99) & 161 & 6 (3.99) & 118 & 7 (10.42) & $46.37 / 17.10$ \\
\hline India & 242 & $7(3.46)$ & 159 & $7(3.46)$ & 83 & $9(7.33)$ & $5.52 / 5.80$ \\
\hline Taiwan & 231 & $8(3.30)$ & 204 & $8(3.30)$ & 27 & 17 (2.39) & $8.23 / 12.37$ \\
\hline Germany & 200 & $9(2.86)$ & 90 & $9(2.86)$ & 110 & $8(9.72)$ & $13.00 / 18.39$ \\
\hline Spain & 182 & $10(2.60)$ & 112 & $10(2.60)$ & 70 & $10(6.18)$ & $8.42 / 10.77$ \\
\hline Italy & 159 & II (2.27) & 96 & II (2.27) & 63 & II (5.57) & $16.71 / 18.57$ \\
\hline UK & 154 & $12(2.20)$ & 8 & $12(2.20)$ & 146 & $6(12.90)$ & $35.75 / 20.93$ \\
\hline Australia & 124 & I3 (I.77) & 66 & I3 (I.77) & 58 & $12(5.12)$ & $22.11 / 11.52$ \\
\hline Sweden & 124 & $13(1.77)$ & 69 & 13 (1.77) & 55 & $13(4.86)$ & $17.16 / 18.29$ \\
\hline Israel & 103 & 15 (I.47) & 55 & 15 (I.47) & 48 & $14(4.24)$ & $27.93 / 29.94$ \\
\hline Singapore & 98 & $16(1.40)$ & 74 & $16(1.40)$ & 24 & $18(2.12)$ & 22.62/I5.7| \\
\hline Switzerland & 84 & $17(1.20)$ & 42 & $17(1.20)$ & 42 & 15 (3.7I) & $16.21 / 26.45$ \\
\hline Russia & 71 & $18(1.02)$ & 55 & $18(1.02)$ & 16 & $22(\mathrm{I} .4 \mathrm{I})$ & $2.73 / 13.75$ \\
\hline The Netherlands & 56 & $19(0.80)$ & 33 & $19(0.80)$ & 23 & $19(2.03)$ & $13.06 / 10.09$ \\
\hline Poland & 54 & $20(0.77)$ & 33 & $20(0.77)$ & 21 & $20(1.86)$ & $3.73 / 24.67$ \\
\hline
\end{tabular}

Abbreviations: CP, international collaborative publications; P, number of publications; IP, independent publications; TCP, citation time per publication. 


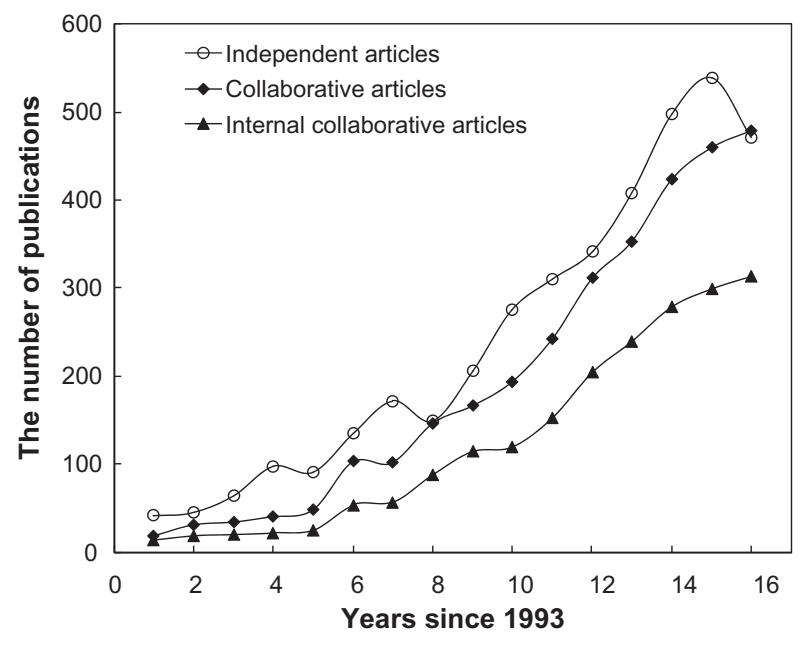

Figure 2 Collaboration share of world articles, internal share of world collaboration articles, and independent articles per year.

greatest quantity of papers, is ranked fifth in international collaborative publications.

Among the 6992 articles with address information, 3842 $(54.95 \%)$ were publications by a single institute, and others were collaborative work, both international (16.19\%) and internal $(28.86 \%)$ collaborations included. Figure 2 shows collaborative, internal collaborative articles and independent articles per year. Independent articles made up the majority of the output each year. Additionally, both collaborative and internal collaborative articles, maintain consistent growth publication rates.

The relationship between cumulative number of publications and publication year during 1993-2008 is shown in Figure 3. A power model can be used to represent the cumulative progress of collaboration, internal collaboration, and independent articles (Equations 3-8):

Collaborative articles:

$\begin{array}{lll}1993-1996 & y=19.18 x^{1.350} & R^{2}=0.999 \\ 1996-2008 & y=2.999 x^{2.492} & R^{2}=0.998\end{array}$

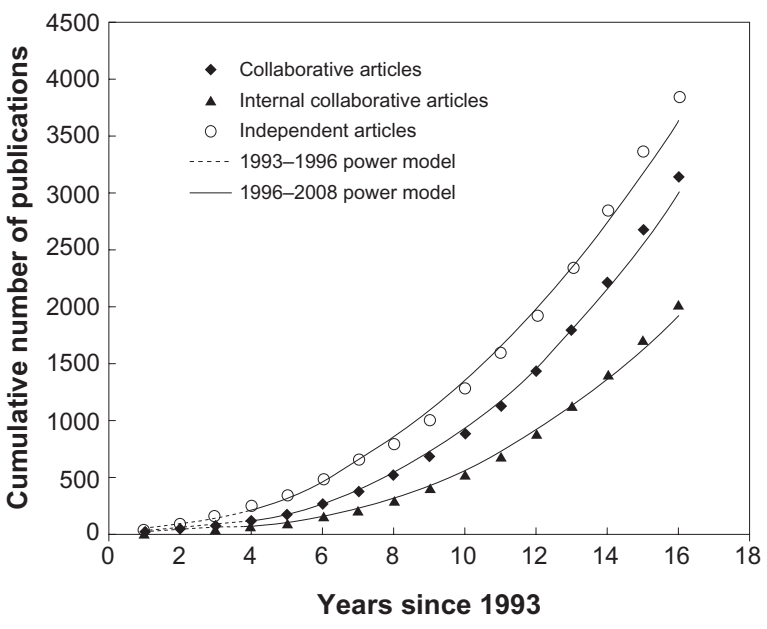

Figure 3 Cumulative number of independent articles and collaborative internal collaborative articles by year.

Internal collaborative articles:

$$
\begin{array}{lll}
1993-1996 & y=14.08 x^{1.209} & R^{2}=0.999 \\
1996-2008 & y=1.366 x^{2.614} & R^{2}=0.997
\end{array}
$$

Independent articles:

$$
\begin{array}{lll}
\text { 1993-1996 } & y=41.20 x^{1.152} & R^{2}=0.995 \\
1996-2008 & y=10.65 x^{2.103} & R^{2}=0.995
\end{array}
$$

During the period from 1996 to 2008, the high coefficient of the power model reveals that the cumulative number of collaborative articles will be equal to the number of independent articles by 2018. Moreover, the internal collaboration share of the collaboration will increase gradually to $67.78 \%$ in 2018 .

\section{Publication patterns: subject categories and journals}

The bibliometric analysis of publications in the field of

\begin{tabular}{|c|c|c|c|c|c|}
\hline Subject categories & $\begin{array}{l}\text { I 993- | } 996 \\
\text { Rank (\%) }\end{array}$ & $\begin{array}{l}\text { I 997-2000 } \\
\text { Rank (\%) }\end{array}$ & $\begin{array}{l}200 \mathrm{I}-2004 \\
\text { Rank (\%) }\end{array}$ & $\begin{array}{l}\text { 2005-2008 } \\
\text { Rank (\%) }\end{array}$ & $\begin{array}{l}\text { I993-2008 } \\
\text { Rank (\%) }\end{array}$ \\
\hline Electrochemistry & I (4I.05) & I (50.26) & I (49.30) & I (45.II) & I (46.82) \\
\hline Materials science, multidisciplinary & $6(7.89)$ & $4(15.18)$ & $3(20.22)$ & $2(26.20)$ & $2(21.96)$ \\
\hline Chemistry, physical & $2(25.00)$ & $2(21.36)$ & $2(2|| 0)$. & $3(21.69)$ & $3(21.65)$ \\
\hline Energy and fuels & $5(12.89)$ & $5(12.15)$ & $4(|5.4|)$ & $4(13.57)$ & $4(13.88)$ \\
\hline Materials science, coatings and films & $3(16.58)$ & $3(19.58)$ & $5(12.49)$ & $5(1|.5|)$ & $5(13.17)$ \\
\hline Physics, condensed matter & $4(16.32)$ & $6(10.47)$ & $6(9.58)$ & $6(9.96)$ & $6(10.26)$ \\
\hline Chemistry, multidisciplinary & $9(3.42)$ & $7(3.56)$ & $7(6.76)$ & $7(8.23)$ & $7(6.90)$ \\
\hline Physics, applied & $7(4.2 I)$ & $10(2.20)$ & $9(3.35)$ & $8(6.30)$ & $8(4.77)$ \\
\hline Metallurgy and metallurgical engineering & $21(1.05)$ & $16(1.47)$ & II (2.53) & $9(5.86)$ & $9(4.03)$ \\
\hline Chemistry, inorganic and nuclear & $14(2.37)$ & $9(2.83)$ & $8(3.84)$ & II (4.38) & $10(3.90)$ \\
\hline
\end{tabular}
lithium ion battery research shows 101 subject categories identified by the Institute of Scientific Information during

Table 5 Top ten subject categories with most publications 
Table 6 Top 15 most published journals on lithium ion battery research

\begin{tabular}{|c|c|c|c|c|c|c|}
\hline Journal & $\begin{array}{l}\text { 1993-1996 } \\
\text { Rank (\%) }\end{array}$ & $\begin{array}{l}\text { I997-2000 } \\
\text { Rank (\%) }\end{array}$ & $\begin{array}{l}200 \mathrm{I}-2004 \\
\operatorname{Rank}(\%)\end{array}$ & $\begin{array}{l}\text { 2005-2008 } \\
\text { Rank (\%) }\end{array}$ & $\begin{array}{l}\text { 1993-2008 } \\
\text { Rank (\%) }\end{array}$ & IF \\
\hline J Power Sources & $3(12.63)$ & $2(10.99)$ & I (I4.92) & I (13.18) & I (I3.37) & 3.477 \\
\hline J Electrochem Soc & I (16.32) & I (19.27) & $2(12.06)$ & $2(10.79)$ & $2(12.6 I)$ & 2.437 \\
\hline Electrochimica Acta & $4(5.53)$ & $4(6.28)$ & $4(5.74)$ & $3(7.10)$ & $3(6.80)$ & 3.078 \\
\hline Solid State Ionics & $2(13.68)$ & $3(8.06)$ & $3(6.76)$ & $6(3.6 I)$ & $4(5.38)$ & 2.425 \\
\hline Electrochem Solid State Lett & - & $5(3.35)$ & $5(4.57)$ & $4(4.7 I)$ & $5(4.23)$ & 2.001 \\
\hline Electrochem Commun & - & II (I.57) & $6(3.69)$ & $5(4.43)$ & $6(3.59)$ & 4.194 \\
\hline Chem Mater & $9(1.58)$ & $7(2.4 I)$ & $8(2.53)$ & $7(2.86)$ & $7(2.63)$ & 5.046 \\
\hline J Phys Chem B & - & $8(2.20)$ & $9(2.38)$ & $9(1.84)$ & $8(1.95)$ & 4.189 \\
\hline Chin J Inorg Chem & - & $81(0.10)$ & $17(0.92)$ & $8(2.28)$ & $9(1.47)$ & 0.532 \\
\hline J Mater Chem & $7(1.84)$ & $9(1.88)$ & $10(2.09)$ & $20(0.88)$ & $10(1.42)$ & 4.646 \\
\hline Electrochemistry & - & $13(1.36)$ & $17(0.92)$ & $25(0.69)$ & II (I.32) & 0.835 \\
\hline Mater Chem Phys & - & $52(0.21)$ & $7(2.67)$ & $12(1.5 \mathrm{I})$ & $12(1.21)$ & 1.799 \\
\hline J Alloys Compd & $16(0.79)$ & $30(0.42)$ & $26(0.53)$ & II (I.7I) & $13(1.14)$ & 1.510 \\
\hline J Solid State Chem & $10(1.32)$ & $10(1.68)$ & $12(1.36)$ & $21(0.80)$ & $14(1.12)$ & 1.910 \\
\hline J Solid State Electrochem & - & $19(0.84)$ & II (I.4I) & $14(1.32)$ & $15(1.07)$ & 1.597 \\
\hline
\end{tabular}

Abbreviation: IF, impact factor.

the last 16 years. The distribution of the top ten subject categories in lithium ion battery research from 1993 to 2008 is shown in Table 5, including the ranking of paper quantities in four periods, ie, 1993-1996, 1997-2000, 2001-2004, and 2005-2008. The result indicates that Electrochemistry has been the leading category during these 16 years. Because lithium-based battery research is highly multidisciplinary, the ranking for the publications of 'Materials Science, Multidisciplinary' and 'Metallurgy and Metallurgical Engineering' improved over time.

In total, 7025 Science Citation Index Expanded publications referring to lithium ion battery research were published in 537 journals, within which $46 \%$ journals contained only one article and $15.27 \%$ journals contained two articles. Table 6 presents the top 15 journals with the most publications in the lithium ion battery research field. 'Journal of Power Sources' published the most lithium ion batteryrelated articles $(939 ; 13.37 \%)$, followed by 'Journal of the Electrochemical Society' (886; 12.61\%), and 'Electrochimica Acta' $(478 ; 6.80 \%)$. Also notable is the improvement in ranking of 'Electrochemistry Communications', 'Journal of Alloys and Compounds', and 'Chinese Journal of Inorganic Chemistry' after 1997.

\section{Research emphasis: words in title and author keywords}

An analysis of title words was performed for a total of 7025 articles over the last 16 years. Prepositions, articles, and conjunctions in titles were excluded from the statistic analysis. Figure 4 presents title words which have appeared in over 200 articles. Specifically, "electrochemical", "synthesis", "properties", "material(s)", "anode", "cathode", "electrolytes", "polymer", "carbon", "characterization", "graphite", "spinel”, "rechargeable", "LiMn $\mathrm{O}_{4}$ ”, "intercalation" and " $\mathrm{LiCoO}_{2}$ " were the most frequently used during the period 1993-2008.

Keywords appeared in the articles referring to lithium ion battery research from 1993 to 2008 were calculated and ranked over the total 16 years of the study and 4-year time periods. A total of 4380 articles, with records that included keywords in the Science Citation Index Expanded database between 1993 and 2008, were calculated in this analysis. There are 7391 keywords listed, of which 5511 (74.56\%) were used once and $834(11.28 \%)$ were used twice. The top 60 author keywords in the last 16 years are shown in Table 7, through which changes in overall research focus can be roughly identified. "lithium-ion battery", "lithium ion battery", "lithium-ion

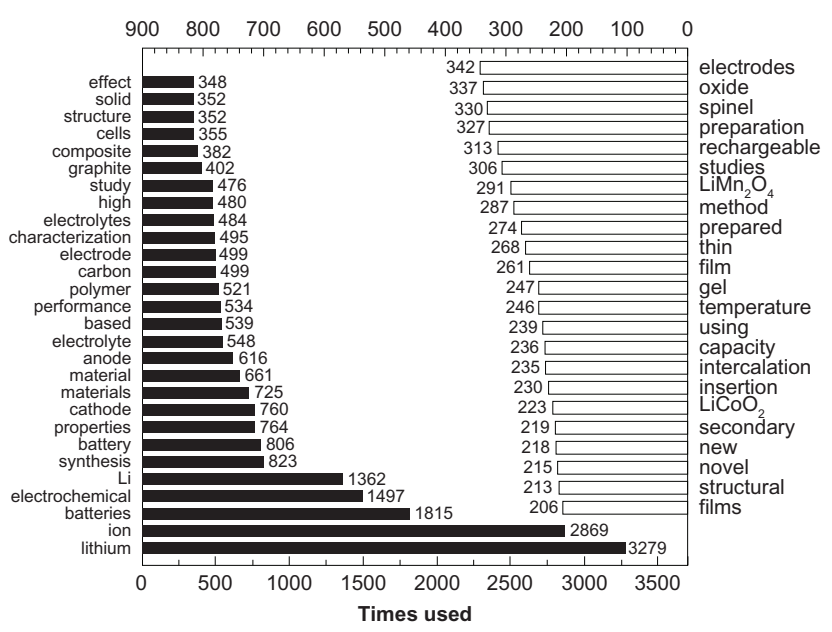

Figure 4 Top 51 title words appearing more than 200 times. 
Table 7 Top 50 keywords used in lithium ion battery research articles

\begin{tabular}{|c|c|c|c|c|c|c|}
\hline \multirow[t]{2}{*}{ Author keywords } & \multirow{2}{*}{$\begin{array}{l}\text { 93-96 } \\
\text { Rank (\%) }\end{array}$} & \multirow{2}{*}{$\begin{array}{l}97-00 \\
\text { Rank (\%) }\end{array}$} & \multirow{2}{*}{$\begin{array}{l}\text { OI-04 } \\
\text { Rank (\%) }\end{array}$} & \multirow{2}{*}{$\begin{array}{l}\text { 05-08 } \\
\text { Rank (\%) }\end{array}$} & \multicolumn{2}{|c|}{$93-08$} \\
\hline & & & & & $\mathbf{P}$ & Rank (\%) \\
\hline lithium-ion battery & $74(0.60)$ & $3(7.36)$ & I (I0.82) & I (II.89) & 465 & I (10.62) \\
\hline lithium ion battery & $74(0.60)$ & I (9.15) & $2(9.09)$ & $2(10.00)$ & 406 & $2(9.27)$ \\
\hline lithium-ion batteries & $5(4.82)$ & $4(7.16)$ & $3(6.91)$ & $3(9.12)$ & 353 & $3(8.06)$ \\
\hline lithium ion batteries & $8(4.22)$ & $28(1.59)$ & $4(6.6 I)$ & $4(8.03)$ & 294 & $4(6.7 I)$ \\
\hline cathode material & $74(0.60)$ & 38 (I.39) & $9(3.76)$ & $5(7.94)$ & 247 & $5(5.64)$ \\
\hline Li-ion battery & - & $28(1.59)$ & $5(4.8 I)$ & $6(6.43)$ & 225 & $6(5.14)$ \\
\hline electrochemical properties & - & 17 (2.39) & $12(3.01)$ & $7(4.79)$ & 166 & $7(3.79)$ \\
\hline Li-ion batteries & - & $12(2.58)$ & $10(3.53)$ & $9(4.45)$ & 166 & $7(3.79)$ \\
\hline anode & $74(0.60)$ & $28(1.59)$ & $14(2.78)$ & $8(4.7 \mathrm{I})$ & 158 & $9(3.61)$ \\
\hline ionic conductivity & $2 I(2.4 I)$ & $10(2.98)$ & $7(3.91)$ & II (3.28) & 149 & $10(3.40)$ \\
\hline Lithium & I (I7.47) & $2(8.35)$ & $16(2.63)$ & $24(1.76)$ & 148 & II (3.38) \\
\hline lithium batteries & $8(4.22)$ & $7(4.37)$ & II (3.46) & $15(2.90)$ & 144 & $12(3.29)$ \\
\hline cathode materials & $74(0.60)$ & $28(1.59)$ & $12(3.01)$ & $10(3.91)$ & 142 & $13(3.24)$ \\
\hline graphite & $3(6.63)$ & $6(4.77)$ & $6(4.06)$ & $20(2.14)$ & 140 & $14(3.20)$ \\
\hline $\mathrm{LiMn}_{2} \mathrm{O}_{4}$ & $74(0.60)$ & $28(1.59)$ & $8(3.83)$ & $16(2.65)$ & 123 & I5 (2.8I) \\
\hline Lithium battery & $5(4.82)$ & $8(3.18)$ & $14(2.78)$ & $17(2.6 \mathrm{I})$ & 123 & I5 (2.8I) \\
\hline cathode & $74(0.60)$ & $22(1.99)$ & $17(2.55)$ & $14(3.19)$ & 121 & $17(2.76)$ \\
\hline anode material & $74(0.60)$ & $28(1.59)$ & $21(2.25)$ & II (3.28) & 117 & I8 (2.67) \\
\hline $\mathrm{LiCoO}_{2}$ & $37(1.20)$ & $12(2.58)$ & $17(2.55)$ & I8 (2.44) & 107 & $19(2.44)$ \\
\hline intercalation & $2(10.84)$ & $5(4.97)$ & $17(2.55)$ & $44(1.01)$ & 101 & $20(2.3 \mathrm{I})$ \\
\hline polymer electrolyte & $4(6.02)$ & $12(2.58)$ & $21(2.25)$ & $24(1.76)$ & 95 & $21(2.17)$ \\
\hline spinel & $15(3.01)$ & $12(2.58)$ & $20(2.48)$ & $27(1.72)$ & 92 & $22(2.10)$ \\
\hline $\mathrm{LiFePO}_{4}$ & - & - & I I 7 (0.45) & II (3.28) & 84 & $23(1.92)$ \\
\hline X-ray diffraction & $23(I .8 I)$ & $23(1.79)$ & $29(1.50)$ & $21(2.02)$ & 80 & $24(1.83)$ \\
\hline electrolyte & $37(1.20)$ & $83(0.60)$ & $23(2.03)$ & $22(1.97)$ & 79 & $25(1.80)$ \\
\hline anode materials & - & $62(0.80)$ & $28(1.73)$ & $24(1.76)$ & 69 & $26(1.58)$ \\
\hline conductivity & $15(3.01)$ & $28(1.59)$ & $24(1.88)$ & $34(1.30)$ & 69 & $26(1.58)$ \\
\hline electrochemical performance & - & $83(0.60)$ & $68(0.75)$ & $19(2.23)$ & 66 & $28(I .5 I)$ \\
\hline battery & $74(0.60)$ & $18(2.19)$ & $29(1.50)$ & $36(1.26)$ & 62 & $29(1.42)$ \\
\hline polymer electrolytes & $5(4.82)$ & $23(1.79)$ & $25(1.80)$ & $6 \mathrm{I}(0.84)$ & 61 & $30(1.39)$ \\
\hline electrochemistry & $37(1.20)$ & $138(0.40)$ & $50(0.90)$ & $23(1.85)$ & 60 & $31(1.37)$ \\
\hline carbon & $12(3.6 \mathrm{I})$ & $18(2.19)$ & $25(1.80)$ & $64(0.76)$ & 59 & $32(1.35)$ \\
\hline cyclic voltammetry & $37(1.20)$ & $62(0.80)$ & $50(0.90)$ & $28(\mathrm{I} .5 \mathrm{I})$ & 54 & $33(1.23)$ \\
\hline lithium ion & $37(1.20)$ & $18(2.19)$ & $50(0.9)$ & $39(1.18)$ & 53 & $34(1.21)$ \\
\hline sol-gel & $74(0.60)$ & $23(1.79)$ & $43(0.98)$ & $36(1.26)$ & 53 & $34(1.21)$ \\
\hline lithium intercalation & $8(4.22)$ & $10(2.98)$ & $32(1.43)$ & $109(0.46)$ & 52 & $36(1.19)$ \\
\hline batteries & $12(3.6 \mathrm{I})$ & $38(1.39)$ & $39(1.05)$ & $44(1.01)$ & 51 & $37(1.16)$ \\
\hline thin film & - & $23(1.79)$ & $43(0.98)$ & $39(1.18)$ & 50 & $38(1.14)$ \\
\hline doping & $23(\mathrm{I} .8 \mathrm{I})$ & $83(0.60)$ & $68(0.75)$ & $30(1.39)$ & 49 & $39(1.12)$ \\
\hline sol-gel method & - & $257(0.20)$ & $34(1.20)$ & $31(1.34)$ & 49 & $39(1.12)$ \\
\hline thermal stability & - & $257(0.20)$ & $29(1.50)$ & $42(1.09)$ & 47 & $4 \mid(1.07)$ \\
\hline coating & - & $138(0.40)$ & $50(0.90)$ & $31(1.34)$ & 46 & $42(1.05)$ \\
\hline lithium manganese oxide & - & $18(2.19)$ & $25(1.80)$ & $109(0.46)$ & 46 & $42(1.05)$ \\
\hline silicon & - & I $38(0.40)$ & $77(0.68)$ & $29(1.47)$ & 46 & $42(1.05)$ \\
\hline surface modification & - & $62(0.80)$ & $43(0.98)$ & $38(1.22)$ & 46 & $42(1.05)$ \\
\hline lithium-ion & - & $8(3.18)$ & $43(0.98)$ & $84(0.59)$ & 43 & $46(0.98)$ \\
\hline capacity & $37(1.20)$ & $44(0.99)$ & $37(1.13)$ & $6 \mathrm{I}(0.84)$ & 42 & $47(0.96)$ \\
\hline thin films & $23(1.81)$ & $83(0.60)$ & $43(0.98)$ & $48(0.97)$ & 42 & $47(0.96)$ \\
\hline gel polymer electrolyte & - & $83(0.60)$ & $37(1.13)$ & $48(0.97)$ & 41 & $49(0.94)$ \\
\hline impedance spectroscopy & $74(0.60)$ & $44(0.99)$ & $33(1.28)$ & $64(0.76)$ & 41 & $49(0.94)$ \\
\hline
\end{tabular}


batteries", "lithium ion batteries", "Li-ion battery", "Li-ion batteries" are the six common author keywords used in lithium ion battery-related articles.

It is worth noticing from Table 7 that the main test methods used in lithium ion battery research during the last 16 years were "X-ray diffraction", "cyclic voltammetry", and "impedance spectroscopy" (also "electrochemical impedance spectroscopy", "AC impedance"). The "electrochemical property(ies)" and "electrochemical performance" of batteries are a constant theme in battery research, and "thermal stability", "capacity", and "conductivity" are the primary concerns for lithium ion rechargeable battery researchers.

Since the emergence of the lithium ion battery, exploration of the best-performing combination of anode-electrolytecathode has been a constant focus in lithium ion battery research. As a result, an immense amount of work has been done on these components, as shown in the following three sections. Analysis of author keywords on these specific topics can serve as an innovative approach for mapping global lithium ion battery research.

\section{Anode electrode materials}

In the early stage of the study period (1993-1996), carbon negative electrodes were frequently explored as a result of their straightforward intercalation of lithium. ${ }^{7}$ Because "graphite" and "carbon" ranked 3 and 12, they were obviously a significant hotspot from 1993 to 1996. As the technology in lithium ion batteries matured, the ranking of "graphite" and "carbon" in the study period decreased. Researchers shifted their interests towards exploiting new anode materials which possessed a larger capacity than graphite. "Silicon", for instance, shows a steady ascent in ranking as shown in Table 7. The number and proportion of articles including "silicon" increased from " $2,0.4 \%$ " in 1997-2000 to " 35 , $1.47 \%$ " in 2005-2008.

\section{Cathode materials}

For cathode materials, the ranking and proportions of data from Table 7 show that " $\mathrm{LiCoO}_{2}$ " "7,8 and " $\mathrm{LiMn}_{2} \mathrm{O}_{4}$ " were the most widely studied materials before 2004. Although the capacity is less than " $\mathrm{LiCoO}_{2}$ ", the "spinel" " $\mathrm{LiMn}_{2} \mathrm{O}_{4}$ " system has an advantage in terms of cost and is comparatively nontoxic. Thus, lots of works were dedicated to improving its limitations in cycling and storage performance..$^{9-11}$

Another development in cathode material research is the adoption of "LiFePO ${ }_{4}$ " ${ }^{12-16}$ The number and proportion of articles referring to " $\mathrm{LiFePO}_{4}$ " have increased rapidly, from "6, 0.45\%" during 2001-2004 to "78, 3.28\%". Owing to the advantage of potentially lower cost and improved safety, this material has increasingly attracted the interest of researchers, in spite of its technical disadvantage of low conductivity.

\section{Electrolyte}

Besides the electrodes, the electrolyte is the third key component of a battery. Since the introduction of polymeric electrolytes in a liquid lithium ion system by Bellcore in $1996,{ }^{17}$ polymer electrolytes have become one of the major hotspots in lithium ion battery research. The number of articles with the keywords "polymer electrolyte" or "polymer electrolytes" has jumped from 20 during 1993-1996 to 147 during 2005-2008.

There are 301 articles referring "polymer electrolyte" in the author keywords, comprised of "polymer electrolyte(s)" (156), "gel polymer electrolyte(s)" (47), "solid polymer electrolyte(s)" (25), "composite polymer electrolyte(s)" (24), "microporous polymer electrolyte" (6), "nanocomposite polymer electrolytes" (4), and other keywords including the same phrases. Generally, we can classify all the polymer electrolyte systems into two broad categories, ie, solid polymer electrolyte and gel polymer electrolyte. In terms of the number of publications, the gel polymer electrolyte has attracted more attention than the solid polymer electrolyte, which may be attributed to its higher ionic conductivity at ambient temperature. ${ }^{18-20}$

\section{Conclusion}

In the present study, global scientific production in the lithium ion battery research field was quantitatively characterized from 1993 to 2008. The results show that the cumulative number of papers increased in the 16-year study period, and that this increasing trend was represented by two power models. Researchers from the three large geographic regions, North America, western Europe, and eastern Asia, contributed most of the publications. The United States was ranked top in collaborative publications in this field. As the leader in absolute quantity of published lithium ion battery-related articles, China needs to improve further the quality of output. Two power models were used to express the cumulative progression of collaboration and internal collaboration, respectively. An immense amount of research has been carried out on the cathode, anode, and electrolyte. Finally, analysis of author keywords and words in the title successfully shows the hotspots in the field of lithium ion battery research, and it is hoped that this may serve to direct and inform future dialog as to the direction of lithium ion battery research. 


\section{Disclosure}

The authors report no conflicts of interest in this work.

\section{References}

1. Murphy DW, Disalvo FJ, Carides JN, Waszczak JV. Topochemical reactions of rutile related structures with lithium. Mater Res Bull. 1978;13:1395-1402.

2. Lazzari M, Scrosati B. A cyclable lithium organic electrolyte cell based on two intercalation electrodes. J Electrochem Soc. 1980;127:773-774.

3. Schalkwijk WV, Scrosati B, editors. Advances in Lithium-Ion Batteries. Heidelberg, Germany: Springer; 2002.

4. Sony Corporation. Sony announces lithium ion polymer battery production in Singapore. Available from: http://www.sony.net/SonyInfo/ News/Press/200708/07-0814E/index.html. Accessed August 13, 2009.

5. Xie S, Zhang J, Ho Y-S. Assessment of world aerosol research trends by bibliometric analysis. Scientometrics. 2008;77:113-130.

6. Ho Y-S. Bibliometric analysis of biosorption technology in water treatment research from 1991 to 2004. Int J Environ Pollut. 2008; $34: 1-13$

7. Wakihara M. Recent developments in lithium ion batteries. Mat Sci Eng R. 2001;33:109-134.

8. Mizushima K, Jones PC, Wiseman PJ, Goodenough JB. LixCoO $(0<\mathrm{x}<-1)$ : A new cathode material for batteries of high-energy density. Mater Res Bull. 1980;15:783-789.

9. Guyomard D, Tarascon JM. High-voltage stable liquid electrolytes for LixMn $\mathrm{O}_{4} /$ Carbon rocking-chair lithium batteries. J Power Sources. 1995;54:92-98.

10. Thackeray MM, David WIF, Bruce PG, Goodenough JB. Lithium insertion into manganese spinels. Mater Res Bull. 1983;18:461-472.
11. Amatucci G, Du Pasquier A, Blyr A, Zheng T, Tarascon JM. The elevated temperature performance of the $\mathrm{LiMn}_{2} \mathrm{O}_{4} / \mathrm{C}$ system: failure and solutions. Electrochim Acta. 1999;45:255-271.

12. Muraliganth T, Murugan AV, Manthiram A. Nanoscale networking of $\mathrm{LiFePO}(4)$ nanorods synthesized by a microwave-solvothermal route with carbon nanotubes for lithium ion batteries. J Mater Chem. 2008;18:5661-5668

13. Maccario M, Croguennec L, Desbat B, Couzi M, Le Cras F, Servant L. Raman and FTIR spectroscopy investigations of carbon-coated Li(x) FePO(4) materials. J Electrochem Soc. 2008;155:A879-A886.

14. Dedryvere R, Maccario M, Croguennec L, Le Cras F, Delmas C, Gonbeau D. X-ray photoelectron spectroscopy investigations of carboncoated LixFePO materials. Chem Mater. 2008;20:7164-7170.

15. Wang JZ, Chou SL, Chen J, et al. Paper-like free-standing polypyrrole and polypyrrole- $\mathrm{LiFePO}_{4}$ composite films for flexible and bendable rechargeable battery. Electrochem Commun. 2008;10:1781-1784.

16. Wang ZL, Sun SR, Xia DG, Chu WS, Zhang S, Wu ZY. Investigation of electronic conductivity and occupancy sites of mo doped into $\mathrm{LiFePO}_{4}$ by ab initio calculation and x-ray absorption spectroscopy. J Phys Chem C. 2008;112:17450-17455.

17. Tarascon JM, Gozdz AS, Schmutz C, Shokoohi F, Warren PC Performance of Bellcore's plastic rechargeable Li-ion batteries. Solid State Ionics. 1996;86-8:49-54.

18. Armand M. The history of polymer electrolytes. Solid State Ionics. 1994;69:309-319.

19. Croce F, Appetecchi GB, Persi L, Scrosati B. Nanocomposite polymer electrolytes for lithium batteries. Nature. 1998;394:456-458.

20. Imrie CT, Ingram MD, McHattie GS. Ion transport in glassy polymer electrolytes. J Phys Chem B. 1999;103:4132-4138.
Reports in Electrochemistry

\section{Publish your work in this journal}

Reports in Electrochemistry is an international, peer-reviewed, open access journal publishing original research, reports, reviews and commentaries on all areas of electrochemistry. The manuscript management system is completely online and includes a very quick and fair

\section{Dovepress}

peer-review system. Visit http://www.dovepress.com/testimonials.php to read real quotes from published authors. 